\title{
Some consequences of stereoscopic depth constancy
}

\author{
HANS WALLACH \\ Swarthmore College, Swarthmore, Pennsylvania 19081 \\ BARBARA GILLAM \\ SUNY College of Optometry, New York, New York 10010 \\ and \\ LEONARD CARDILLO \\ Swarthmore College, Swarthmore, Pennsylvania 19081
}

\begin{abstract}
Retinal disparity decreases in proportion to the square of the distance of the corresponding objective depth interval from the eyes. Up to a distance of $2 \mathrm{~m}$, stereoscopic depth perception compensates well for this decrease in disparity with observation distance; for a given disparity, experienced depth increases approximately in proportion to the square of the observation distance. When disparities are artificially produced, by anaglyphs or vectograms, or by spectacles, they decrease only in proportion to the first power of the observation distance. The same is true when the Pulfrich effect gives rise to equivalents of disparity. Depth perception, however, compensates for the normal disparity loss. As a result, there should be a net gain in perceived depth approximately in proportion to the first power of observation distance. When perceived depth caused by horizontal magnification in one eye or by the Pulfrich effect was measured, it was found to increase approximately in proportion to observation distance.
\end{abstract}

Stereoscopic depth constancy is a compensation process that corrects for the dependence of the magnitude of retinal disparity on observation distance and, within limits, makes depth perception veridical. Ono and Comerford (1977) have provided a review of experimental and theoretical work on this topic. Our purpose is to supplement their review by describing and illustrating some interesting consequences of stereoscopic depth constancy. These consequences result from its peculiar quantitative nature. The retinal disparity that corresponds to a depth interval-i.e., to a difference between the distances of two points in space from the eyes-is inversely proportional to the square of the distance of the depth interval from the eyes. If stereoscopic depth constancy is complete, this dependence of disparity on distance is compensated for, so that disparity of a certain amount will cause a perceived depth interval that is proportional to the square of the observation distance. While the geometry of disparity is such that disparity decreases in proportion to the square of the observation distance, the compensation process causes perceived depth to increase in proportion to the square of that distance. In the compensation

This work was supported in part by Grant BNS75-19095 A01 from the National Science Foundation to Swarthmore College, Hans Wallach, principal investigator. We are most grateful to Martha Teghtsoonian for her help in writing this report. process, it is "registered" distance-the distance given to the nervous system by the available distance cues-that operates: If registered distance differs from physical distance, constancy will lag and perceived depth will not be veridical. Stereoscopic depth constancy may be compared with size constancy. In size perception, the size of the retinal image that represents the size of the corresponding object is inversely proportional to the distance of the object rather than to the square of the distance. Size constancy compensates for this effect of distance on image size; an image of a certain size will cause a perceived size that is proportional to the object's registered distance.

There are two ways in which one can measure the degree to which these constancies perform their compensations. One can present a certain object size or depth interval at different distances from the eyes and measure to what degree perceived size and perceived depth remain constant. Such measurements were performed by Fried (1974) for depth perception, and when distance cues were sufficient there was good correspondence between perceived depth and objective depth up to a distance of $2 \mathrm{~m}$. Or one can keep the object or the depth interval at the same objective distance and alter only the distance cues with which they are given. This has the advantage that image sizes and disparities remain the same for the conditions whose perceptual results are being 
compared. Wallach and Zuckerman (1963) obtained size and depth estimates for a wire form at a distance of $133 \mathrm{~cm}$ under two conditions, in natural viewing or when an arrangement of mirrors and lenses caused the eyes to be converged and accommodated for half the objective distance. Since convergence and accommodation were the only distance cues in operation, the arrangement made the registered distance of the wire form half of $133 \mathrm{~cm}$. Complete size and stereoscopic depth constancies would predict that, with registered distance so diminished but with image size and disparities unaltered, perceived size would be half and perceived depth one-quarter of what they were in natural viewing. The results actually obtained fell about $25 \%$ short of the predicted effect. This lag in constancy, however, was the same for size and for depth. Since it is known that, for distances of $133 \mathrm{~cm}$ and shorter, size constancy is complete when distance cues are adequate, the lags that were here measured must be ascribed to the inadequate distance cues provided by accommodation and convergence. Thus, the results are compatible with Fried's finding that stereoscopic depth constancy can be complete for distances up to $2 \mathrm{~m}$.

An effect analogous to that reported by Wallach and Zuckerman (1963) is seen when one uses binoculars. Binocular telescopes diminish registered distances in two ways. Along with all visual angles, they increase convergence angles in proportion to their power, and increased convergence angles mean proportionally shorter distances. Secondly, along with all retinal images, telescopes make the images of familiar objects larger in proportion to their power. Because the sizes of the images of familiar objects serve as distance cues-the distances they represent are inversely proportional to these sizes-the enlarged images caused by the telescopes again mean shorter distances. As a result, the depth one sees through the glasses is greatly diminished. The effect of binoculars, however, differs from the effect of the mirror and lens arrangement of the experiment just cited; with binoculars, size perception remains veridical. This is because binoculars enlarge retinal image sizes in the same proportion as the distances that are represented by the altered distance cues are diminished. For the same reason, depth is not diminished as much as in the experiment by Wallach and Zuckerman. Six-power binoculars, e.g., by magnifying the retinal images of familiar objects and convergence angles sixfold, cause distances as registered by the nervous system to be diminished by a factor of six and, if disparities were unaltered, perceived depth should be diminished by a factor of six squared. But disparities do not remain unaltered. By magnifying all retinal images by a factor of six, the glasses enlarge disparities sixfold also. Since the sixfold reduction of registered distance would diminish perceived depth by a factor of six squared if disparities remained the same, and since the glasses enlarge disparities sixfold, perceived depth is diminished by a factor of six-compared to the depth one would perceived if one were six times nearer to the scene and looked without the glasses. In general, perceived depth should be diminished by binocular magnification in proportion to its power. This statement is, however, based on the assumption that stereoscopic depth constancy operates undiminished at distances larger than $2 \mathrm{~m}$. However that may be, every time someone looks through binoculars and observes that depth appears diminished, he confirms that stereoscopic depth constancy compensates for the effect of distance on disparity at a rate higher than the first power of the registered distance.

The other consequences of stereoscopic depth constancy to be discussed have this in common: Compensation for registered distance occurs in connection with disparities that are not produced by objective depth intervals. We know of three different ways of producing disparities or their equivalents in the absence of objective depth intervals-stereoscopically, by spectacles, and by means of the Pulfrich effect.

\section{Stereoscopically Produced Disparities}

Vectograms and anaglyphs are stereoscopic arrangements where the left-eye and the right-eye picture are presented superposed on each other in the same picture plane. In anaglyphs, the two pictures are rendered in different colors, and each is prevented from forming images in one of the eyes by color filters of the same chromaticity. Homologous left- and right-eye points or lines lie next to each other, and their horizontal distances from each other are the pictorial disparities which replace real depth intervals. Such pictorial disparities are outward projections, so to speak, of the disparities that the three-dimensional scene represented in the anaglyph would produce in the two eyes if it were really given. The retinal disparities that correspond to these pictorial disparities vary, like the retinal images of all other pictorial features, inversely with the distance of the anaglyph from the eyes, while disparities produced by real depth intervals would vary inversely with the square of the distance of the scene from the eyes. Thus, if stereoscopic depth constancy operates without a lag and distance cues are adequate to provide for veridical registered distances, perceived depth should increase in proportion to the objective distance of the anaglyph from the eyes.

Wallach and Zuckerman (1963) had subjects view the same anaglyph from a distance of $45 \mathrm{~cm}$ and again from a distance of $90 \mathrm{~cm}$. The subjects made two estimates for a particular depth interval in the 
scene they saw by adjusting calipers, one estimate for each viewing distance. The mean caliper settings were 2.04 and $3.45 \mathrm{~cm}$, and the mean estimate of depth perceived with the large viewing distance was 1.68 times as large as the other mean estimate. That ratio would have been expected to be 2 , if stereoscopic depth constancy had operated optimally. The lag was probably due to the fact that only accommodation and convergence were available as cues for distance; when Wallach, Frey, and Bode (1972) obtained size estimates of objects at distances of 33.3 and $66.7 \mathrm{~cm}$ under similar limitations, their results implied a ratio of registered distances of 1.64 instead of 2.

In vectograms, the left- and right-eye pictures are made to reach the appropriate eyes by using polarized light in conjunction with polarizing filters. This technique is employed in three-dimensional movies. Vectograms, too, represent depth in the form of pictorial disparities and are therefore subject to the same dependence on observation distance. Ideally, the depth that is perceived increases in proportion to the registered observation distance. That accounts (1) for the large changes in depth one perceives when, with the polarizing glasses in place, one walks down the aisle toward the screen, and (2) for the fact that perceived depth is realistic only at a limited range of distances from the screen.

\section{Disparities Produced by Spectacles}

Analogous variations of perceived depth that depends on registered distance should be expected to occur when disparities are caused by spectacles. The simplest of such spectacles, a meridional size lens placed in front of one eye, causes minor magnification of the horizontal dimension in that eye (Ames, 1946). Such magnification will increase horizontal distances on the retina of that eye, and the resulting differences between homologous horizontal separations in the two eyes are the disparities that the spectacles produce. These disparities will cause a patterned surface in the subject's frontal plane to appear slanted away from him on the side of the magnifier.

As in the case of pictorial disparities, the disparities caused by monocular magnification will decrease only in proportion to the observation distance. The magnifier lengthens the projections of horizontal separations in one eye by a certain factor and thereby produces disparities for which there is no objective depth. Perceived depth would therefore increase with the square of the distance of the frontal surface whose slant is observed were it not for the fact that projections of the horizontal separations in both eyes decrease in proportion to the distance of that surface. Because they result from magnification, disparities are proportional to the sizes of the horizontal separations and will also decrease in proportion to the observation distance. With disparities decreasing in proportion to observation distance and perceived depth increasing with the square of this distance, the net result should be an increase of perceived depth in proportion to the observation distance. Our experiment was to test whether the perceived slant caused by monocular horizontal magnification behaves accordingly.

The apparent slant of a frontal surface caused by placing a meridional size lens in front of one eye was measured with Gillam's (1968) successful method of measuring aniseikonia. The surface was a vertical glass plate whose upper two-thirds was covered with a nearly random dot pattern. Black dots, approximately $1.5 \mathrm{~mm}$ in diameter, were scattered over the surface, with 64 dots placed randomly in each of $905 \times 5 \mathrm{~cm}$ areas. The framed glass plate was symmetrically mounted on a vertical shaft so that it could be turned to any slant. It was seen against the luminous surface of a glow box behind it. The luminance measured at the glass was $630 \mathrm{fL}$.

A pulley from a toy construction set, $6.4 \mathrm{~cm}$ in diameter, served as a comparison stimulus. It was mounted with its rim on the lengthened vertical shaft of a reduction gear motor so that the shaft formed the extension of one of its diameters. The pulley was placed $6 \mathrm{~cm}$ in front of the lower, dot-free region of the glass plate. The subject controlled the slant of the pulley by operating the reduction gear motor. He sat with his head in a chinrest at one of three distances from the glass plate. The visual field of each of the eyes of a subject was restricted by screens and a septum to a binocular region of $32^{\circ}$ in the horizontal dimension and $16^{\circ}$ in the vertical. The lower part of the field of the eye that was equipped with the lens was blocked so that the subject saw the pulley only with the unaided eye. The perception of the pulley's slant was mediated in the case of larger slant angles by deformations of the pulley and its textured surface. Smaller slant angles could be perceived monocularly because the pulley had threedimensional features, a bulging rim with a V-shaped slot and a bushing at the center. To bring out these features, the pulley was illuminated obliquely from the side by a special beam.

The subject had to reproduce the perceived slant of the dotted plate by setting the pulley to a matching slant. There were three distances between his eyes and the center of the dotted plate, 40,70 , and $100 \mathrm{~cm}$. Three meridional size lenses were used; they provided $2 \%, 5 \%$, and $8 \%$ magnification. The nine combinations of distance and magnification were presented twice to each subject. Before the lenses were used, the subject was given practice in matching the dotted plate's orientation in depth with the slant of the pulley. The experimenter repeatedly set the dotted plate to one of three slants and the subject had to 
Table 1

Mean Tangents of the Matches for the Apparent Slant Angles of a Frontal Plane as a Function of Horizontal Magnification in One Eye and of Viewing Distance

\begin{tabular}{|c|c|c|c|c|c|c|}
\hline \multirow{3}{*}{$\begin{array}{c}\text { Distance } \\
\text { (in Centimeters) }\end{array}$} & \multicolumn{6}{|c|}{ Magnification } \\
\hline & \multicolumn{2}{|c|}{$2 \%$} & \multicolumn{2}{|c|}{$5 \%$} & \multicolumn{2}{|c|}{$8 \%$} \\
\hline & $\tan$ & $\tan / \mathrm{d} \times 100$ & $\tan$ & $\tan / \mathrm{d} \times 100$ & $\tan$ & $\tan / \mathrm{d} \times 100$ \\
\hline 40 & .081 & .324 & 242 & .606 & .362 & .904 \\
\hline 70 & .179 & .255 & 461 & .659 & .563 & .804 \\
\hline 100 & .359 & .359 & 544 & .544 & .785 & .785 \\
\hline Average & & .313 & & .603 & & .831 \\
\hline
\end{tabular}

make a match. Thereafter the measurements of the lens-induced apparent slants were made with the glass plate fixed in the subject's frontal plane. At the start of each match, the experimenter put the pulley in the frontal position. Bracketing was permitted and was freely used by both subjects who served in the experiment.

The mean slant matches for each distance at the three horizontal magnifications are shown in Table 1 , with the slant angles changed into tangents. Table 1 shows that the perceived slant increases with magnification and with observation distance. How accurately the mean matches of perceived slant increase in proportion to observation distance is shown by the quotients of mean slant over distance that are given in the columns headed $\tan / \mathrm{d} \times 100$. If the matches of perceived slant had increased exactly in proportion to the observation distance, the three quotients within a column should have been the same. This is approximately the case for the $5 \%$ and $8 \%$ magnification. The averages of the quotients in each column show the effect of magnification as such. The theoretical values for these quotients are .3 for $2 \%, .73$ for $5 \%$, and 1.14 for $8 \%$ magnification. ${ }^{1}$ A comparison shows a considerable shortfall for the $8 \%$ magnification. This shortfall is probably caused by the conflicting information about slant provided by stereoscopic cues, on the one hand, and the appearance of the dot pattern, on the other. As given by stereoscopic cues, the slant angles at the distances of 70 and $100 \mathrm{~cm}$ should have been $38.6^{\circ}$ and $48.7^{\circ}$. At such slants, a distortion of the dot pattern would be noticeable, but the pattern, being actually in the subject's frontal plane, is given without such a distortion.

\section{Depth Produced by the Pulfrich Effect}

There is still another way to evoke stereoscopic vision without presenting real depth intervals-by means of the Pulfrich effect. (For a detailed description of the effect, see Lit, 1949.) This effect produces an equivalent of retinal disparity by making use of the fact that the rate of neural transmission from retinal receptors to the visual cortex varies with the intensity of stimulation. Suppose a target moves horizontally from right to left in a frontoparallel plane while the subject fixates a stationary vertical rod located in the same plane. When a filter over the subject's left eye diminishes intensity of stimulation and thereby the rate of transmission from that eye, the filter-equipped eye will see the target always to the right of the location in which it is seen with the right eye. The two monocular locations constitute a crossed disparity that results in the moving target's being seen nearer than the fixated rod. When the target moves from left to right, the same transmission delay causes the equivalent of an uncrossed disparity and it will be perceived beyond the fixated rod. It is easy to see that the magnitude of the disparity varies with the motion velocity. Hence, a simple harmonic reciprocating motion causes an apparent elliptical path of the moving target. It is, of course, the velocity of the target image on the retina, not the target's physical velocity, that matters here, and that velocity changes also when the target distance from the eye changes. Since the projection of the moving target on the retina is being displaced there with a velocity that is inversely proportional to the distance of the motion path from the eyes, the disparity produced by a certain difference in the rate of transmission will decrease in proportion to that distance. If stereoscopic depth constancy operates in connection with the Pulfrich effect so that the depth that results from a given disparity increases with the square of the registered observation distance, the net result should again be an increase in perceived depth proportional to an increase in distance. We tested for such a result by obtaining measurements of the Pulfrich effect at three different distances.

A vertical rod was made to move horizontally back and forth in the subject's frontoparallel plane with simple harmonic motion. Above its path and in the same plane was a horizontal scale of vertical lines. The lower end of the scale line that was located above the center of the rod's motion path served as fixation mark. The subject held a filter in front of his left eye to diminish the intensity of stimulation there. As a consequence, the rod moving from right to left 
appeared to be nearer to him than the scale. The subject gave estimates of the apparent depth between the scale and the rod in the center part of its path by adjusting outside calipers to represent that depth interval.

The moving rod was $6.4 \mathrm{~mm}$ wide and ended $10 \mathrm{~mm}$ below the edge of the scale, which consisted of lines $2 \mathrm{~mm}$ wide and $12.5 \mathrm{~mm}$ apart. The rod's motion path was $33.8 \mathrm{~cm}$ long and one cycle of its motion took $5 \mathrm{sec}$. A black screen $56 \mathrm{~cm}$ in front of the rod's path hid its lower end and the device that made it move. The upper edge of the screen was horizontal and the end of the rod that was visible above it was $9 \mathrm{~cm}$ long and was seen against a backilluminated translucent screen. The scale was well illuminated so that its brightness matched that of the translucent screen. The subject observed the rod's motion binocularly, fixating the designated mark, while holding over his left eye a round neutral density filter that was rated to pass $6.25 \%$ of the light. Depth estimates were given at three viewing distances, 50,100 , and $150 \mathrm{~cm}$.

The following procedure served to assess the individual subject's use of the calipers in estimating depth. Each subject made estimates of a number of real depth intervals presented in random order. The rod used in the Pulfrich presentation was placed at different distances in front of the scale, and the subject had to make a caliper setting that represented the depth interval between the rod and the plane of the scale. The objective distances were $.5,1,2,4,8$, and $10 \mathrm{~cm}$. All estimates were made from a viewing distance of $100 \mathrm{~cm}$. Each subject's estimates of depth were plotted as a function of real depth, and the best fitting regression line was computed; this was used subsequently to convert his Pulfrich estimates to values derived from his estimates of real depth.

The order of the three viewing distances was varied so that each distance occurred in every order position. Half of our 12 subjects gave estimates of the real depth intervals before the Pulfrich estimates, and for the other half, this order was reversed. Paid undergraduates served as subjects.

The means of the Pulfrich depth estimates made at the three viewing distances, together with their standard deviations, are presented in Table 2 . The

Table 2

Means and Standard Deviations of Estimates of the Pulfrich Effects at Three Observation Distances

\begin{tabular}{|c|c|c|c|c|c|c|}
\hline & \multicolumn{6}{|c|}{ Distance (in Centimeters) } \\
\hline & \multicolumn{2}{|c|}{50} & \multicolumn{2}{|c|}{100} & \multicolumn{2}{|c|}{150} \\
\hline & Mean & SD & Mean & SD & Mean & $\mathrm{SD}$ \\
\hline Caliper Settings & 3.57 & 1.18 & 6.18 & 2.20 & 9.70 & 3.57 \\
\hline Converted Estimates & 4.03 & 1.42 & 7.00 & 1.85 & 11.28 & 3.11 \\
\hline
\end{tabular}

means of the actual estimates are listed on the first row and the means of the converted estimates in the second row. Tukey's test for two mean comparisons showed the differences between the means of the converted estimates to be significant. For 50 vs. $100 \mathrm{~cm}$, $q(22)=6.42, p<.01$, and for 100 vs. $150 \mathrm{~cm}$, $\mathrm{q}(22)=9.38, \mathrm{p}<.01$. The ratios between the means were 1.74 (for 50 - and $100-\mathrm{cm}$ distances) and 2.8 (for 50- and $150-\mathrm{cm}$ distances) and fell somewhat short of the values of 2.0 and 3.0 that would be expected if the Pulfrich effect increased in proportion to observation distance.

\section{Discussion}

Pictorially presented disparities, disparities produced by monocular magnification in the horizontal dimension, and the equivalents of disparity caused by the Pulfrich effect all decrease with the first power of observation distance only. Depth perception, however, applies the normal compensation for the decrease of disparity with distance to these artificial conditions. Since perceived depth varies with the square of the observation distance, an overcompensation occurs here. As a result, perceived depth increases with observation distance, ideally in proportion to its first power. Previous measurements and our own experiments showed this to be approximately true.

In none of the experiments here discussed did perceived depth increase accurately in proportion to observation distance. Increases were always somewhat smaller. and implied incomplete constancy. Yet, Fried (19/4) had obtained complete constancy for the distance range within which distances were varied in these experiments. Such differences are probably a function of the adequacy of the distance cues that were available under the different experimental conditions.

Stereoscopic depth constancy is concerned with the magnitude of perceived depth interevals. It plays no role when thresholds of stereoscopic depth are measured, because they should be directly dependent on the angular sizes of the disparities. (See also Lit \& Finn, 1976.) Because the perception of the magnitude of a depth interval is dependent not only on disparity but also on the observation distance, its accuracy is quite low. Cues for observation distance are not plentiful and usually not exact, and since perceived depth is dependent on registered distance squared, the error introduced by this dependence may be quite large.

Ono and Comerford (1977) did not raise the question as to whether stereoscopic depth constancy is learned. It can be argued that it is, because it has been shown that the relation between disparity and the magnitude of perceived depth can be rapidly modified by adaptation. Wallach, Moore, and 
Davidson (1963) obtained mean changes in perceived depth that ranged up to $20 \%$ by exposing subjects to rotating wire forms observed through a disparityenhancing device. This adaptation effect altered stereoscopic depth perception in general, for it was found to transfer to differently shaped objects.

\section{REFERENCES}

Amrs, A., Jr. Binocular vision as affected by relations between uniocular stimulus-patterns in commonplace environments. American Journal of Psychology, 1946, 59, 333-357.

Frifi, A. H. Convergence as a cue to distance. (Doctoral dissertation, New School for Social Research, 1973). Dissertation Abstracts International, 1974, 34, 3247B.

Gil.tam, B. J. Perception of slant when perspective and stereopsis conflict: Experiments with aniseikonic lenses. Journal of Experimental Psychologv, 1968, 78, 299-305.

Lir, A. The magnitude of the Pulfrich stereophenomenon as a function of binocular differences of intensity at various levels of illumination. American Journal of Psychology, 1949, 62, 159-181.
LIT, A., \& FINN, J. P. Variability of depth-discrimination thresholds as a function of observation distance. Journal of the Optical Society of America, 1976, 66, 740-742.

OGLE, K. N. Researches in binocular vision. New York: Hafner, 1950

Ono, H., \& Comerford. J. Stereoscopic depth constancy. In W. Epstein (Ed.), Stability and constancy in visual perception. New York: Wiley-Interscience Publication, 1977.

Wallach, H., Frey, K. J., \& Bone, K. A. The nature of adaptation in distance perception based on oculomotor cues. Perception \& Psychophysics, 1972, 11, 110-116.

Wallach, H., Moore, M., \& Davidson, L. Modification of stereoscopic depth perception. American Journal of Psychology, 1963, 76, 191-204.

Wallach, H., \& Zuckerman, C. The constancy of stereoscopic depth. American Journal of Psychology, 1963, 76, 404-412.

\section{NOTE}

1. The theoretical values were computed by a method designed by Ogle (1950, p. 162).

(Received for publication September 25, 1978; revision accepted July 17, 1979.) 